\title{
Subcutaneous emphysema of the leg: Could be a fatal condition
}

\author{
Mahmoud A. Hafez \\ The Orthopaedic Department, October 6 University, Cairo, Egypt \\ Email: mhafez@msn.com
}

Received 1 May 2012; revised 8 June 2012; accepted 3 July 2012

\section{PATIENT AND METHODS}

A 70-year-old woman referred to the Emergency Department by her family doctor as a possible septic arthritis or fracture of the left hip. She has had increasing pain in her left leg for 3 weeks and recalled a minor fall 5 days earlier followed by inability to weight bear. Incidental diagnosis of asymptomatic kidney stone had been made six months prior with no urinary symptoms or diabetes.

On presentation, the patient was cachectic, pale and apathetic. Blood pressure was 70/51 $\mathrm{mmHg}$, pulse rate $110 / \mathrm{min}$, temperature $36.2^{\circ} \mathrm{C}$. Abdomen was soft with normal bowel sounds but mild tenderness on the left renal angle. Rectal examination was unremarkable. The left thigh and leg were swollen with redness and tenderness over the groin and lateral aspect of the hip. The striking feature was crepitus felt under the skin, indicating subcutaneous emphysema along the lateral aspect of the hip, thigh, calf and down to the ankle. Hip joint was only painful at the extreme range of motion.

Radiography showed a left renal stone and gas in the soft tissue planes of the left retroperitoneal space (Figure 1). The gas was extending to the left hip region and down to the left lower thigh but there was no bony or joint abnormality (Figure 2). WBC was $36,600 / \mathrm{ml}$, random

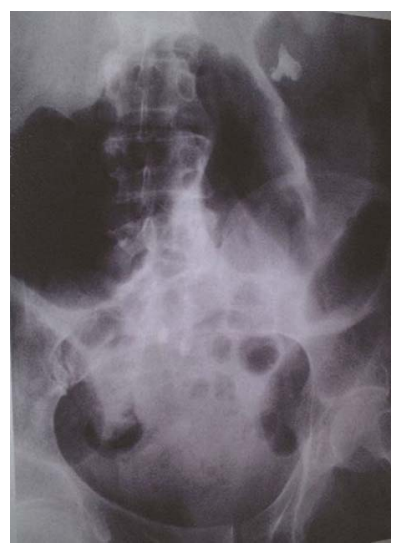

Figure 1. X-ray of the abdomen showing a left renal stone and gas in the soft tissue planes of the left retroperitoneal space. 


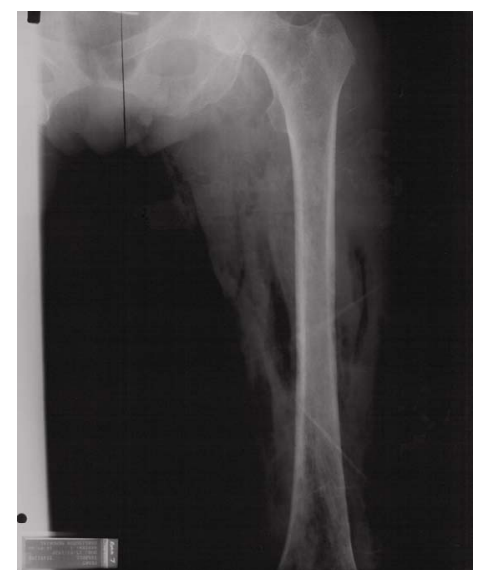

Figure 2. X-ray of the left femur showing gas in the soft tissue planes of the left hip region and whole thigh with no bony or joint abnormality.

plasma glucose $5.8 \mathrm{mmol} / \mathrm{l}$, urea $29.9 \mathrm{mmol} / \mathrm{l}$ and creatinine $113 \mathrm{mmol} / \mathrm{l}$.

Owing to the fact that the patient presented with signs of septic shock at evening time, a decision was made for urgent incision and drainage with no delay for further investigations. Fluid and antibiotic therapy was promptly commenced. Incisions for drainage were made over the point of maximum swelling and fluctuation in the left groin, lateral and medial aspects of the thigh and medial aspects of the leg. Exploration revealed gas in the subcutaneous tissues with large amounts of thick white pus and tissue necrosis that was sent for culture and histopathology. The hip joint felt normal, but the pus extended into the retroperitoneal space up to the left kidney and a loin incision drained a large volume of pus. Though patient was admitted to the Intensive Care Unit and appropriate support was given, she deteriorated and succumbed two days later.

\section{RESULT}

Pus culture revealed Escherichia coli and Bacteroides fragilis. Urine and blood culture showed no growth. Histopathology reported acute non-specific inflammation with necrosis of fat, muscles and fascia. The post-mortem reported a $4 \mathrm{~cm}$ abscess in the lower pole of left kidney and a stag-horn calculus enveloped with greenish-yellow pus in the lower calyx. The abscess had extended to the retroperitoneal, peripelvic tissues, outer wall of left hip and thigh and to the calf with inflammation and marked necrosis.

\section{DISCUSSION}

Subcutaneous emphysema of the leg is a recognised but rare complication of gastrointestinal perforation, where the gas originates directly from the perforated viscus. In our case the leg emphysema was a presenting sign of a silent staghorn calculus in non-diabetic patient. This represents a new cause and mechanism for the formation of leg emphysema. The diagnosis was difficult as the patient was initially referred with possible septic arthritis of the left hip and on arrival to hospital, gas gangrene was suspected. She had no urinary symptoms and there was a lack of abdominal signs. Review of English literature revealed that such case has not been reported before.

Surgeons should consider different sources of gas formation; trauma, gastrointestinal perforation or non-lostridial gas-forming infection (particularly in diabetics). Trauma, without infection, has been recognised as a source $\mathrm{f}$ gas formation [2].

Retroperitoneal infections can spread into the thigh, through the psoas muscle, leading to the formation of psoas abscesses. Altemeier and Alexander [3] studied 189 cases of retroperitoneal abscesses. Only one of their cases showed subcutaneous emphysema of left thigh and pelvis secondary to a ruptured carcinoma of the colon.

Perforation or fistula of gastrointestinal tract has been reported by several authors [4-7] as a source of subcutaneous emphysema and cellulitis of the lower limb. The majority of the reported cases caused a diagnostic dilemma.

Urinary pathology may indirectly cause emphysema of the thigh through a fistula into the gastrointestinal tract. Gow [8] reported a case of reno-inguinal fistula in a diabetic patient who had a periureteric abscess that perforated into the caecum and produced a swelling and crepitus in the right groin. Possibility of benign causes should be borne in mind to prevent unnecessary amputations [9].

Non-clostridial gas-forming infections may occur particularly in diabetics where the gas (carbon dioxide) is formed by fermentation of glucose by organisms such as bacteroides, anaerobic streptococci and coliform. Clifford and Katz [10] reported two cases of subcutaneous emphysema in diabetic patients following renal infections that spread into medial aspect of right thigh in one patient and to the chest wall in the other patient. Both patients had abdominal symptoms and signs pointing to the diagnosis of perinephric abscess. In our case, the patient was not diabetic and had no signs to suggest abdominal pathology.

\section{CONCLUSION}

Subcutaneous emphysema of the leg may represent a late and fatal stage of dissemination of renal infection that could originate from asymptomatic stag-horn calculus. Awareness of such condition is vital to ensure early detection of the primary source and appropriate surgical management. 


\section{REFERENCES}

[1] Sadek, A.-R., Blake, H. and Mehta, A. (2011) Emphysematous cystitis with clinical subcutaneous emphysema. International Journal of Emergency Medicine, 4, 26. doi:10.1186/1865-1380-4-26

[2] Filler, R.M., Griscom, N.T. and Papps A. (1968) Posttraumatic crepitation falsely suggesting gas gangrene. The New England Journal of Medicine, 278, 758-761. doi:10.1056/NEJM196804042781403

[3] Altemeier, W.A. and Alexander, J.W. (1961) Retroperitoneal abscess. Archives of Surgery, 83, 512-524. doi:10.1001/archsurg.1961.01300160024004

[4] Lee, K.-B., Moon, E.-S., Jung, S.-T. and Seo, H.-Y. (2004) Subcutaneous emphysema mimicking gas gangrene following perforation of the rectum: A case report. Journal of Korean Medical Science, 19, 756-758. doi:10.3346/jkms.2004.19.5.756

[5] Highton, L., Clover, J. and Critchley, P. (2009) Necrotising fasciitis of the thigh secondary to a perforated rectal cancer. Journal of Plastic, Reconstructive \& Aesthetic Surgery, 62, e17-e19. doi:10.1016/j.bjps.2007.08.031

[6] Linscheid, R.L., Kelly, P.J. and Symmonds, R.E. (1963) Emphysematous cellulitis of the hip and thigh resulting from an enteric fistula. The Journal of Bone \& Joint Surgery, 45, 1691-1697.

[7] Jager, G.J., Joosten, F., Vd Berkmortel, F.W. and Salemans, J.M.J.I. (1995) Subcutaneous emphysema of the lower extremity secondary to a perforated oesophageal carcinoma. Abdominal Imaging, 20, 23-25. doi:10.1007/BF00199637

[8] Gow, J.G. (1951) A case of reno-inguinal fistula due to periureteric abscess. British Journal of Surgery, 39, 91-92. doi:10.1002/bjs.18003915322

[9] Fox, A., Sheick, H., Ekwobi, C. and Ho-Asjoe, M. (2007) Benign surgical emphysema of the hand and upper limb: Gas is not always gangrene-A report of two cases. Emergency Medicine Journal, 24, 798-799. doi:10.1136/emj.2007.046755

[10] Clifford, N.J. and Katz, I. (1962) Subcutaneous emphysema complicating renal infection by gas-forming coliform bacteria: A report of two cases in diabetic patients. The New England Journal of Medicine, 266, 437-439. doi:10.1056/NEJM196203012660904 